\title{
Nasal Carriage of Staphylococcus aureus among Healthy School Children in Kathmandu Valley
}

\author{
Dev Raj Joshi, Suraj Narayan Shrestha, Rajdeep Bomjan and Kamal Poudel \\ National College, Lainchour, Kathmandu \\ e-mail: joshi_devraj@hotmail.com
}

\begin{abstract}
Staphylococcus aureus remains one of the most frequently isolated pathogens in both community and hospital practices. Methicillin resistant S. aureus (MRSA) continues to be a major cause of serious infections in the community worldwide. This study was undertaken to determine the prevalence of $S$. aureus and MRSA in school children in Kathmandu valley and, also to evaluate the antibiotic susceptibility pattern of the isolates. Identification of the $S$. aureus was confirmed by standard microbiological methods and the antibiotic susceptibility testing was performed by disk diffusion method according to the Clinical Laboratory Standards Institution (CLSI). Of the 324 school children who participated in the study, $38(11.8 \%)$ had S. aureus isolated from nasal swabs. The potential risk factors for the S. aureus carriage showed the antibiotic usage within past 4 weeks to be associated with the rate of the nasal carriage (p value 0.000 ). Out of 38, two (5.2\%) isolates were MRSA. One of the MRSA strains was also resistant to Cloxacillin. Erythromycin and Cloxacillin resistance was present in 3 (7.9\%) isolates. Resistance to two or more antibiotics was noted in 2 $(5.3 \%)$ isolates. However, no strains were resistant to Vancomycin. The results of this study indicated that the carriage of MRSA exists among young healthy school children who lack traditional risk factors for MRSA.
\end{abstract}

Key words: community acquired MRSA, Staphylococcus aureus, nasal carriage, school children

\section{Introduction}

Staphylococcus aureus colonizes large proportion of human populations, besides being a major human pathogen (Nilsson \& Ripa 2006). The anterior nares have been shown to be the main reservoir of $S$. aureus in adults and children (Cole et al. 2001). Nasal carriage of $S$. aureus has demonstrated to be a significant risk factor for nosocomial and community acquired infections in variety of populations (von Eiff et al. 2001). Infections caused by Methicillin or Oxacillin resistant S.aureus (MRSA) is mainly nosocomial and increasingly reported from many countries worldwide (King et al. 2006).

MRSA was first reported in 1961 and recognized as a nosocomial pathogen by late 1960s. The known MRSA risk factors include recent surgery or hospitalization, residence in a long-term care facility, presence of a per-cutaneous device or indwelling catheter of recent dialysis (Brumfitt \& Hamilton-Miller 1989). In 1980s MRSA infections were reported in persons who lacked traditional MRSA risk factors. These infections appeared to be acquired in the community and are now known as community acquired MRSA (CA-MRSA) infections. These infections have been reported worldwide (CDC 2003). Outbreaks have occurred in many settings and among different populations (Graham et al. 2006). Incidence of MRSA continues to increase with emergence of CA MRSA in the developing countries as well and can be found in many settings meaning that in the future we will see younger people carrying the bacteria (Davis et al. 2007).

The nasal carriers of $S$. aureus among school children in Kathmandu valley was reported $24.5 \%$ by Joshi et al. (2003). However, studies examining possible risk factors for the nasal carriage status and CA MRSA are noticeably absent. This study was conducted to determine the prevalence of $S$. aureus and MRSA in school children in Kathmandu valley and to evaluate the antibiotic susceptibility pattern of the isolates.

\section{Materials and Methods}

A cross-sectional descriptive study was conducted among school children studying at public and private schools namely Shanti Vidya Griha Secondary School, Lainchaur, and National Model Science School, Gongabu, in the urban settings of Kathmandu valley from July to December 2007. Written questionnaires concerning demographics and medical history 
(antibiotic usage in the past 4 weeks, presence of respiratory infection, and household number) were completed as per the information provided by the students. Informed consent was obtained from teachers and the students.

All nasal samples were collected from 324 students of the above schools, belonging to class 3-7, aged 5-16 years; boys:167 and girls: 157 . Specimens for the culture were collected from the anterior nares with sterile cotton swab, placed in peptone water and then transported to and processed in microbiological laboratory of National College, Lainchour within 4 hours. All the collected swab samples were inoculated in Mannitol Salt Agar (MSA) and incubated at $37^{\circ} \mathrm{C}$ for 24 hours. Isolates of $S$. aureus were identified by standard microbiological methods and confirmed by different biochemical tests as catalase and coagulase tests (Baird 1996).

Antibiotic susceptibility test of the isolates against different antibiotics (Hi-media) such as Oxacillin (1 $\mathrm{mcg} / \mathrm{disc})$, Erythromycin (15mcg/disc), Gentamicin (30 mcg/disc), Vancomycin (30 mcg/disc), Cloxacillin (10 mcg/disc), and Tetracycline $(30 \mathrm{mcg} / \mathrm{disc})$ were determined by disk diffusion method according to the Clinical Laboratory Standards Institution (CLSI 2006).

Statistical package for the social sciences (SPSS) for windows (version 13.0; SPSS, Chicago, Ill., USA) software was used for the statistical analysis of the data. Frequency and percentage were presented for categorical data. Fischer's exact test was applied to determine potential factors associated with $S$. aureus nasal carriage. The level of significance was set at 0.05 using the two tailed method.

\section{Results}

A total of 324 school children with 167 (51.5\%) boys and $157(48.5 \%)$ girls were included in the study. Overall nasal carriage of $S$. aureus in this study population was $11.8 \%$, and out of 38 isolates, $5.2 \%$ in two children was reported MRSA. Both the children were having antibiotics usage within the past 4 weeks. One of the MRSA strains was also resistant to Cloxacillin.

The statistical analysis of the potential risk factors for the $S$. aureus carriage showed antibiotic usage within past 4 weeks to be associated with the rate of the nasal carriage, whereas no association was found between nasal carriage and sex, having a respiratory infection now, and household number (Table 1).

Of the 38 strains of $S$. aureus isolated from the nasal swabs, the antibiotic sensitivity pattern showed 3 (7.9\%) isolates were resistant against Erythromycin and Cloxacillin. However, all strains were found to be sensitive towards Vancomycin and Gentamicin. Resistance to two or more antibiotics was noted in 2 $(5.3 \%)$ isolates. Two of the strains were resistant to two antibiotics; one strain resistant with the Erythromycin and Tetracycline and the other with the Cloxacillin and Oxacillin respectively.

Table 1. Potential risk factors for $S$. aureus nasal carriage among school children

\begin{tabular}{lllll}
\hline \multicolumn{1}{c}{ Factors } & & Positive No. (\%) & Negative No. (\%) & P value \\
\hline Sex & Boy & $18(10.8)$ & $149(89.2)$ & 0.548 \\
& Girl & $20(12.7)$ & $137(87.3)$ & \\
Antibiotic usage in past 4 weeks & Absent & $29(9.5)$ & $275(90.5)$ & 0.000 \\
& Present & $9(45.0)$ & $11(55.0)$ & \\
Having a respiratory infection now & Absent & $35(13.0)$ & $234(87.0)$ & 0.165 \\
& Present & $3(5.5)$ & $52(94.5)$ & \\
Household number & $<4$ & $4(6.9)$ & $54(93.1)$ & 0.263 \\
& $\geq 4$ & $34(12.8)$ & $232(87.2)$ & \\
\hline
\end{tabular}


Antibiotic susceptibility testing of the nasal isolates found 2 isolates resistant to Oxacillin, 2 isolates resistant to Tetracycline, 3 isolated resistant to Erythromycin, and 3 isolates resistant to Cloxacillin (Table 2).

Table 2. Antibiotic sensitivity pattern of 38 strains of $S$. aureus isolated from nasal carriers

\begin{tabular}{lllc}
\hline Antibiotics & Sensitive No. $(\%)$ & Intermediate No. $(\%)$ & Resistant No. $(\%)$ \\
\hline Cloxacillin & $30(79)$ & $5(13.1)$ & $3(7.9)$ \\
Erythromycin & $25(65.8)$ & $10(26.3)$ & $3(7.9)$ \\
Gentamicin & $38(100)$ & - & - \\
Oxacillin & $36(94.8)$ & - & $2(5.2)$ \\
Tetracycline & $36(94.8)$ & - & $2(5.2)$ \\
Vancomycin & $38(100)$ & - & - \\
\hline
\end{tabular}

\section{Discussion}

S. aureus is one of the most common causes of both community- and hospital-acquired infections of skin, surgical sites, blood, and the lower respiratory tract (Lowy 1998). The anterior nares are the most consistent locations from which $S$. aureus can be isolated. Although this colonization is a normal process, it may be a source for invasive infection (Graham et al. 2006). In this study, $11.7 \%$ of the healthy school children were found to be the carriers of $S$. aureus. This rate was similar to other studies which have been conducted on the same age groups as reported by Lo et al. (2007) and Creech et al. (2005). Similar findings were reported by Joshi et al. (2003) as the nasal carriage rates $(24.5 \%)$ for S. aureus. However, globally such rates have been reported to vary form 18 to $50 \%$ in different populations and the carriage of $S$. aureus in the nose appears to play a key role in the epidemiology and pathogenesis of infection (Kluytmans et al. 1997).

The MRSA rate was found to be $5.2 \%$ among the $S$. aureus carriers. Our finding was lower than the findings of Lo et al. (2007) and Creech et al. (2005) whereas, higher than the finding of Hussain et al. (2001). These variable results in the prevalence of MRSA were reflections of the local endemicity, sanitary standard, environmental conditions, timing and seasonal differences in the design of the work and personal hygiene.

Though the higher proportions of girls $(12.7 \%)$ of the subjects were found to be the carriers of $S$. aureus than boys $(10.8 \%)$, there is no correlation between the gender and the carriage of $S$. aureus. In similar study conducted by Joshi et al. (2003), similar proportion of boys and girls were found to be the carriers of $S$. aureus. The difference in the carriage pattern could be due to the difference in study population of different age groups.
In our study, the risk factors of antibiotic usage in past 4 weeks were found to be associated with the rate of $S$. aureus nasal carriage among school children. This might probably be an identifiable risk factor for MRSA acquisition. It is reported that the use of antibiotics generally changes the rate of $S$. aureus nasal carriage pattern due to the increased antibiotic pressure by indiscriminate and empirical use of these drugs (Kluytmans et al. 1997).

In the study, erythromycin resistance was observed to be $7.9 \%$ against the organism. This corroborates with the finding of Creech et al. (2005) and Dietrich et al. (2004). Oxacillin and Tetracycline resistance were observed $5.2 \%$ each. These indicate the frequency of antimicrobial resistance pattern among $S$. aureus carriers with no exposure to the hospital systems.

The findings of this study showed that the carriage of MRSA exists among young healthy school children who lack traditional risk factors for MRSA. Our knowledge of community-acquired MRSA epidemiology is incomplete, which adds to the challenge of controlling infection by community-acquired MRSA, so, continuing surveillance is needed to more accurately assess the prevalence, geographic distribution and epidemiology of community-acquired infection and to develop strategies that will improve therapy and control the spread.

\section{Acknowledgement}

We wish to extend our sincere thanks to National College, Lainchour, for providing the Microbiology Laboratory facilities needed in accomplishing this study. We are thankful to Shanti Vidhya Griha Secondary School, Lainchour and National Model Science School, Gongabu for their co-operation during the collection of samples. 


\section{References}

Baird, D. 1996. Staphylococcus: Cluster forming gram positive cocci. In: Mackie and MaCartny Practical Medical Microbiology (14 ${ }^{\text {th }}$ Edition) (Eds. J. G. Collee, A. G., Fraser, B. P Marmion, and A. Simmons), Churchill Livingstone, pp. 245-61.

Brumfitt, W. and J. Hamilton-Miller. 1989. Methicillin-resistant Staphylococcus aureus. N. Engl. J. Med. 320:1188-96.

Center for Disease Control and Prevention (CDC). 2003. Methicillin-resistant Staphylococcus aureus in correctional facilities-Georgia, Texas, and California, 2001-2003. MMWR Morb. Mortal. Wkly Rep. 52:992-6.

Clinical Laboratory Standards Institute (CLSI). 2006. Performance of standards for antimicrobial susceptibility testing. 16th informational supplement. M 100-S16. Wayne, Pa.

Cole, A.M., S. Tahk, A. Oren et al. 2001. Determinants of Staphylococcus aureus nasal carriage. Clin. Diagn. Lab. Immunol. 8:1064-1069.

Creech, C.B., D.S. Kernodle, A. Alsentzer et al. 2005. Increasing rates of nasal carriage of methicillin resistant Staphylococcus aureus in healthy children. Pediatr. Infect. Dis. J. 24:617-621.

Davis, S.L. M.B. Perri, S.M. Donabedian et al. 2007. Epidemiology and outcomes of community-associated methicillin-resistant Staphylococcus aureus infection.

J. Clin. Microbiol. 45(6): 1705 - 1711.

Dietrich, D.W., D.B. Auld and L.A. Mermel. 2004. Community-acquired methicillin-resistant Staphylococcus aureus in Southern New England children. Pediatrics 113(4):347-352.

Graham III, P.L., S.X. Lin, and E.L. Larson. 2006. A U.S. population-based survey of Staphylococcus aureus colonization. Ann. Intern. Med. 144:318-325.
Hussaiin, F. M., S. Boyle-Vavra and R. S. Daum. 2001. Community acquired methicillin-resistant Staphylococcus aureus colonization in healthy children attending an outpatient pediatric clinic. Pediatr. Infect. Dis. J. 20:763-767.

Joshi S. R., B. Shrestha and S. R. Basnyat. 2003. Nasal carriage of Stapylococcus aureus, related knowledge and hygienic status in healthy school children. $J$. Nepal Health Res. Council 1(3):20-25.

King, M. D., J.H. Bianca, F.W. Yun, V.K. Ekaterina, M.R. Susan and H. M. Blumberg. 2006. Emergence of community-acquired methicillin-resistant Staphylococcus aureus USA 300 clone as the predominant cause of skin and soft-tissue infections. Annals. 144: 309-317.

Kluytmans, J., A. van Belkum and H. Verbrugh. 1997. Nasal carriage of Staphylococcus aureus: epidemiology, underlying mechanisms, and associated risks. Clin. Microbiol. Rev. 10:505-520.

Lo, W., W. Lin, M. Tseng et al. 2007. Nasal carriage of a single clone of community-acquired methicillinresistant Staphylococcus aureus among Kindergarten attendees in northern Taiwan. BMC Infect. Dis. 7:51-56

Lowy, F. D. 1998. Staphylococcus aureus infections. N. Engl. J. Med. 339:520-532.

Nilsson, P. and T. Ripa. 2006. Staphylococcus aureus throat colonization is more frequent than colonization in the anterior nares. J. Clin. Microbiol. 44:33343339.

von Eiff, C., K. Becker, K. Machka, H. Stammer and G. Peters. 2001. Nasal carriage as a source of Staphylococcus aureus. N. Engl. J. Med. 344(18):1399-1401. 